\title{
Eine kleine Revolution in der Chromatographie-Verschraubungstechnik
}

Die bisher verwendeten einteiligen Kunststoffschrauben (mit integriertem Klemmring) haben bei allen Handhabungsvorteilen einen entscheidenden Nachteil. Beim Anziehen wird die Kapillare verdreht und durch Abrieb können zusätzlich Partikel entstehen. Die entsprechenden negativen Folgen in Injektionsventilen und Durchflusszellen sind ausreichend bekannt.

Den Ingenieuren von VICI Valco ist dies nicht verborgen geblieben.

Die Neuentwicklung vereinigt die Vorteile von einteiligen Fittings mit denen von separaten Schrauben und Klemmringen.

Ein sich drehender Klemmring ist in die Spitze der Schraube eingelassen und dreht sich so lange, bis die Verbindung

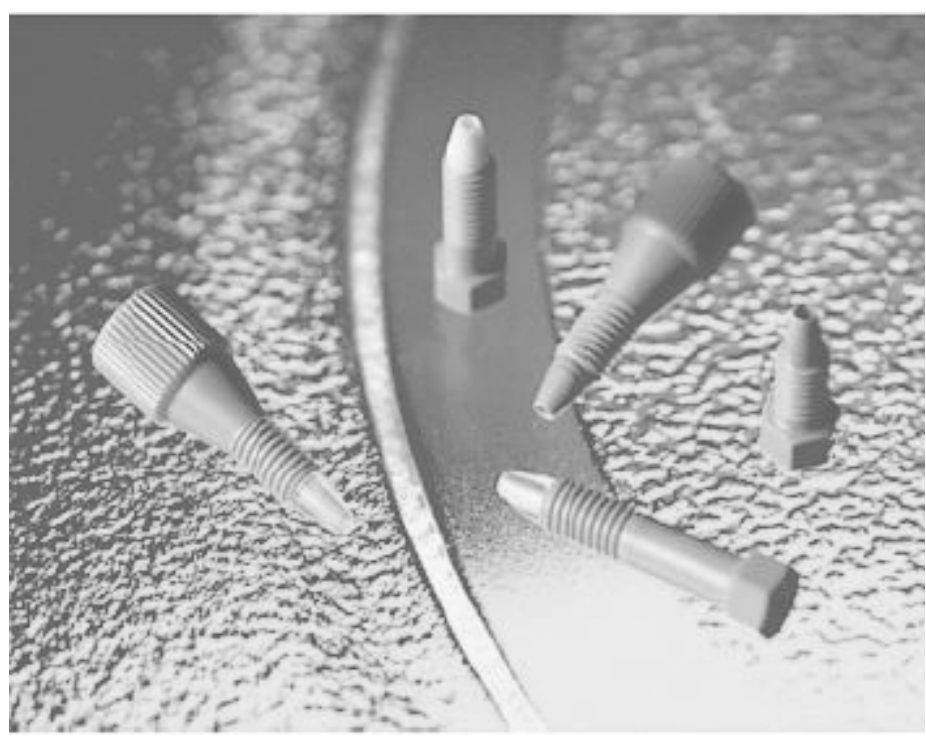

mit der Kapillare dicht ist. Mit diesem revolutionären Design können als weiterer Vorteil unterschiedliche Materialien für den Klemmring verwendet wer- den. Für eine lange Lebensdauer des Klemmrings kommt zum Beispiel glasfaserverstärktes PEEK zum Einsatz. Mit den neuen Produkten können

sowohl 1/16" Kunststoff- sowie Stahlkapillaren verschraubt werden. Die Verbindungen sind abhängig vom verwendeten Klemmring- und Kapillarmaterial druckstabil und dicht bis 600 bar (60.0 MPa).

Lieferbar sind kurze bis lange Sechskantschrauben sowie eine „Fingertight"-Version. Die folgenden Klemmring-Materialien sind als Standard verfügbar: CTFE, PEEK und glass-filled PEEK. Spezielle Materialien wie FEP, PFA und glass-filled PTFE sind auf Anfrage ebenso erhältlich.

- OmniLab AG

Untere Bahnhofstrasse 14 8932 Mettmenstetten

Telefon 0447685656

Fax 0447682321

E-Mail: omnilab@omnilab.ch

Leserdienst Nr. 2

\section{Sprüherstarrung}

Moderne Möglichkeiten in der pharmazeutischen Forschung liefern komplexe, schwer wasserlösliche Wirkstoffe. Die Verabreichung dieser Wirkstoffe stellt daher auch neue Herausforderungen an die Wissenschaft.

Die Verkapselung oder Immobilisierung von aktiven Wirkstoffen, nicht mischbaren Phasen, Biokatalysatoren oder sensitiven Substanzen ist ein wachsender Trend für den optimale Einsatz einer Substanz. Vielfältigste Methoden zur Herstellung von Mikrokapseln stehen zur Verfügung. Je nach Anforderungen bzgl. Partikelgrösse, chemische und physikalische Stabilität, Abbaukinetik etc. werden die Prozesse evaluiert.
Die Sprühtrocknung ist eine bekannte und weit verbreitete Methode, um Partikel bzw. Mikrokapseln herzustellen. Eine verwandte Methode, aber mit anderen «thermodynamischen Vorzeichen» ist die Sprüherstarrung: Eine Schmelze wird in einen kalten Gasstrom verdüst, sodass die Tropfen zu Partikeln erstarren und abgeschieden werden können. Diese Methode wird bereits erfolgreich in der kosmetischen, pharmazeutischen und Nahrungsmittelindustrie eingesetzt. Typische Trägerstoffe sind Fette, Wachse, Stearate oder Polyethylenglykole.

Bis anhin war die Forschung und Prozessentwicklung mit Sprüherstarrung erst im grösseren Massstab durchführbar. Mit

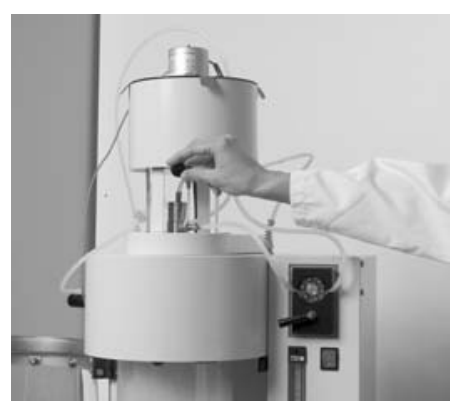

Feindosierventil. Ein zusätzlicher, externer Thermostat ist nicht notwendig. Der Temperaturbereich für Schmelzen liegt zwischen $30^{\circ}$ und $70^{\circ} \mathrm{C}$.

Bei tiefem Schmelzpunkt wird zusätzlich ein Luftvorkühler eingesetzt, der die Eingangsluft für eine schnelle und wirksame $\mathrm{Er}$ starrung auf $-5^{\circ} \mathrm{C}$ herunterkühlt.

einem neuen System von Büchi Labortechnik ist nun erstmals auch ein System im Labormassstab verfügbar. Der etablierte Sprühtrockner «Mini Spray Dryer B-290» ist so erweitert worden, dass auch Sprüherstarrungen durchgeführt werden können. Das Zubehör besteht aus einem thermostatisierbaren Gefäss für den Feed, einer Heizung mit Regelung und einem 


\section{Präzisionsglassäulen für Nieder- und Mitteldruckchromatographie}

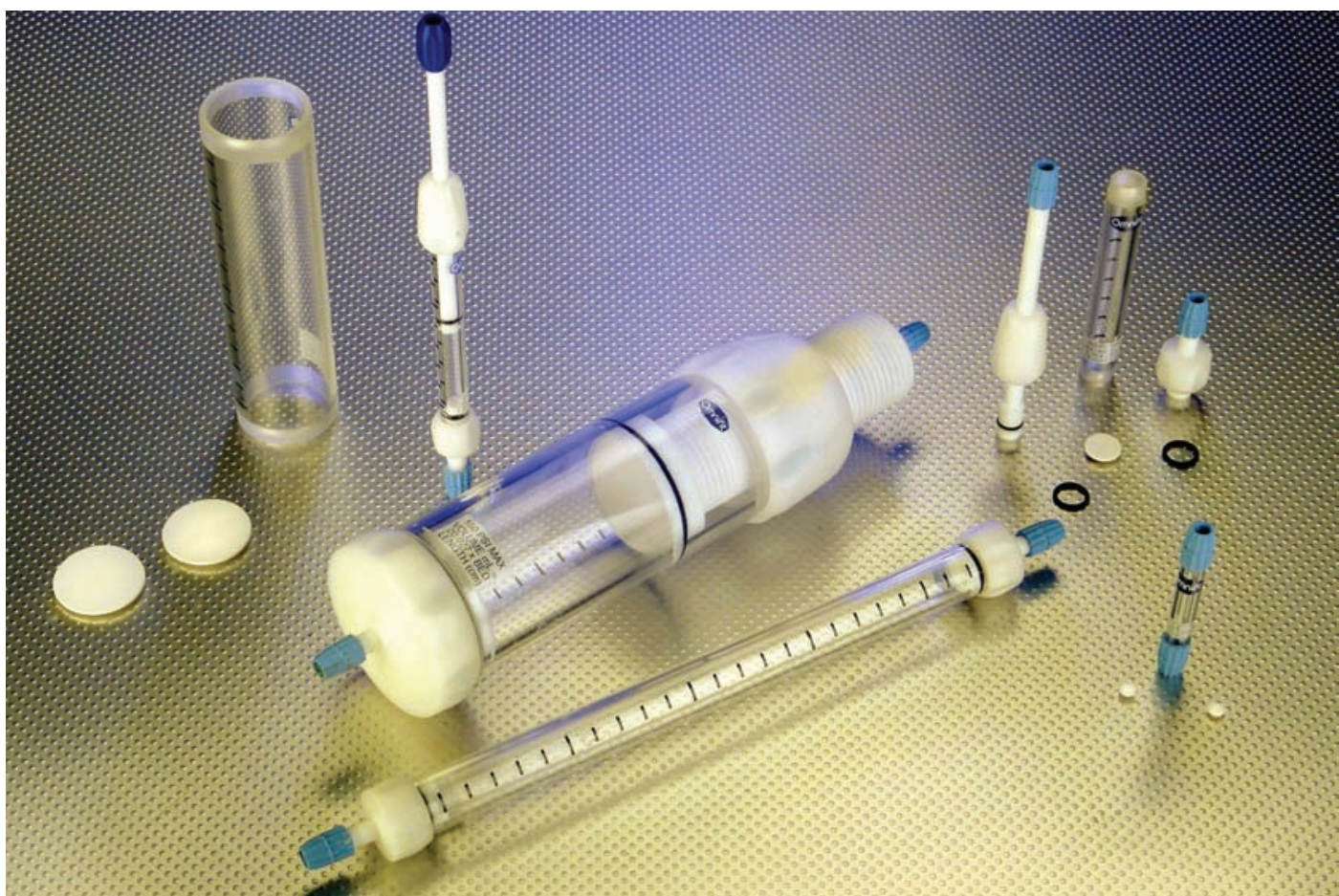

Omnifit's Sortiment an GlasChromatographiesäulen, erhältlich exklusiv von msscientific Chromatographie-Handel $\mathrm{GmbH}$, bietet eine komplette Lösung für Nieder- und Mitteldruckanwendungen. Die Säulen sind robust, einfach in der Handhabung, chemisch hochresistent, biokompatibel, und können, je nach Grösse, mit bis zu 80 bar betrieben werden.

Aus präzisions-gebohrtem Borosilicatglas und mit benetzten Teilen aus inertem PTFE versehen, halten die Säulen extremen Bedingungen stand. Ihr Totvolumen ist minimal, so dass der Fluss gleichmässig bleibt und Kontaminierungen ausgeschlossen sind.
Die Endstücke sind ergonomisch geformt und lassen sich schnell und einfach justieren (bis $\mathrm{zu} 80 \mathrm{~mm}$ Bettlängenjustage). Jede Säule wird komplett montiert geliefert. Zum Lieferumfang gehören alle notwendigen Fittings und diverse Fritten. Weitere Fritten mit Porengrössen bis zu $10 \mu \mathrm{m}$ sind erhältlich. Die vor- montierten PE-Fritten lassen sich jederzeit durch PTFE ersetzen.

Folgende Innendurchmesser sind erhältlich: $3 \mathrm{~mm}, 6,6 \mathrm{~mm}, 10$ $\mathrm{mm}, 15 \mathrm{~mm}, 25 \mathrm{~mm}, 35 \mathrm{~mm}$ und $50,5 \mathrm{~mm}$. Je nach Durchmesser werden die Säulen mit einer Länge von $50 \mathrm{~mm}$ bis $1000 \mathrm{~mm}$ angeboten. Das Angebot wird durch eine Fülle von Zubehörteilen abgerundet. Leser-Anfragen bei:

- Bio-Chem Valve / Omnifit 2 College Park Coldhams Lane Cambridge CB1 3HD United Kingdom Tel.: +44 (0) 1223416642 Fax: +44 (0) 1223416787 E-Mail: sales@omnifit.com Website: www.omnifit.com

Leserdienst Nr. 4

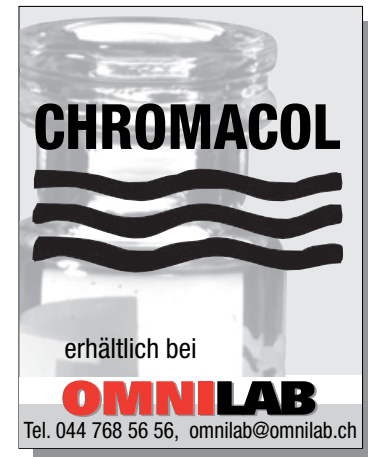

\section{Leserdienst «CHIMIA-REPORT»}

\section{CHIMIA-Leserdienst Heft 7-8/2005}

Chimia-Report (Talon 3 Monate gültig)

Ich bitte um Unterlagen zu den angekreuzten Kennziffern:

$\begin{array}{rrrrrrrrrr}1 & 2 & 3 & 4 & 5 & 6 & 7 & 8 & 9 & 10 \\ 11 & 12 & 13 & 14 & 15 & 16 & 17 & 18 & 19 & 20 \\ 21 & 22 & 23 & 24 & 25 & 26 & 27 & 28 & 29 & 30\end{array}$

Name

Firma

Strasse

$\mathrm{PLZ/Ort}$

Datum

Unterschrift
Die Beiträge der Rubrik "CHIMIA-REPORT» sind mit einer Kennziffer markiert.

Wenn Sie zu einem oder mehreren der auf diese Weise gekennzeichneten Informationsangebote zusätzliche Auskünfte erhalten möchten, empfiehlt sich als einfachster und billigster Weg:

1. Entsprechende Nummer(n) auf dem nebenstehenden Leserdienst-Talon anzeichnen

\section{Absender angeben}

3. Talon an untenstehende Adresse faxen oder einsenden

Ihre Anfragen werden sofort an die einzelnen Firmen weitergeleitet, die Ihnen die gewünschten Unterlagen gerne zur Verfügung stellen werden. Wir freuen uns, wenn Sie unseren Leserdienst benutzen!

\section{KRETZ AG}

CHIMIA-Leserdienst

Postfach

$\mathrm{CH}-8706$ Feldmeilen

Telefon 044 · 92550 60, Fax 044 • 9255077 
In der Prozesskälte sind kältetechnische Anlagen eng mit den Produktionsabläufen verzahnt. Kälteanlagenbauer müssen deshalb kundenspezifische Anforderungen wie Normen und Spezifikationen genau beachten und hochwertige Komponenten einsetzen. Ein Anwendungsgebiet, das grosses Know-how erfordert.

Prozesskälte, also die Bereitstellung von Kälte für industrielle Herstellungsverfahren, wird hauptsächlich für die Verflüssigung und Reinigung von Stoffströmen eingesetzt. Durch Kühlung können Gase gezielt kondensiert und damit abgeschieden werden. Darüber hinaus findet Prozesskälte bei der Kühlung chemischer Reaktionen Verwendung.

In jüngster Zeit kommen in der Prozesskälte verstärkt natürliche Kältemittel zum Einsatz. Ammoniak nimmt dabei mit über 40 Prozent den grössten Anteil ein, gefolgt von Kohlendioxid und Kohlenwasserstoffen. Die grosse Bedeutung des Ammoniaks verwundert nicht: «Vor dem Hintergrund des Kohlendioxid-Emissionshandels ist für viele Anwender die Energie-effizienz das wichtigste Kriterium bei der Auswahl eines geeigneten Kältemittels», erläutert Georges Hoeterickx, Vorstandsmitglied von eurammon, der europäischen Initiative für natürliche Kältemittel. «Da Ammoniak als effizientes Kältemittel anerkannt ist, entscheiden sich viele Unternehmen für dieses traditionsreiche, natürliche Kältemittel.»

\section{Fernkältezentrale für wirtschaftliche Kälte}

So beauftragte der Chemiekonzern Bayer die Kältespezialisten von York mit dem Bau einer Ammoniak-Kälteanlage. Anlass zur Zusammenarbeit war die Erweiterung der Fernkältezentralen im Hauptwerk Leverkusen - mit rund 600 Gebäuden auf über drei Quadratkilometern eine der grössten Produktionsstätten der Welt. Hier stellt Bayer Pharmazeutika, Farbstoffe, Kautschuk, Polyurethane sowie organische und anorganische Produkte her.

Die in den verfahrenstechnischen Prozessen anfallende Wärme wird über eine Fernkälteanlage mit mehreren Kälte-

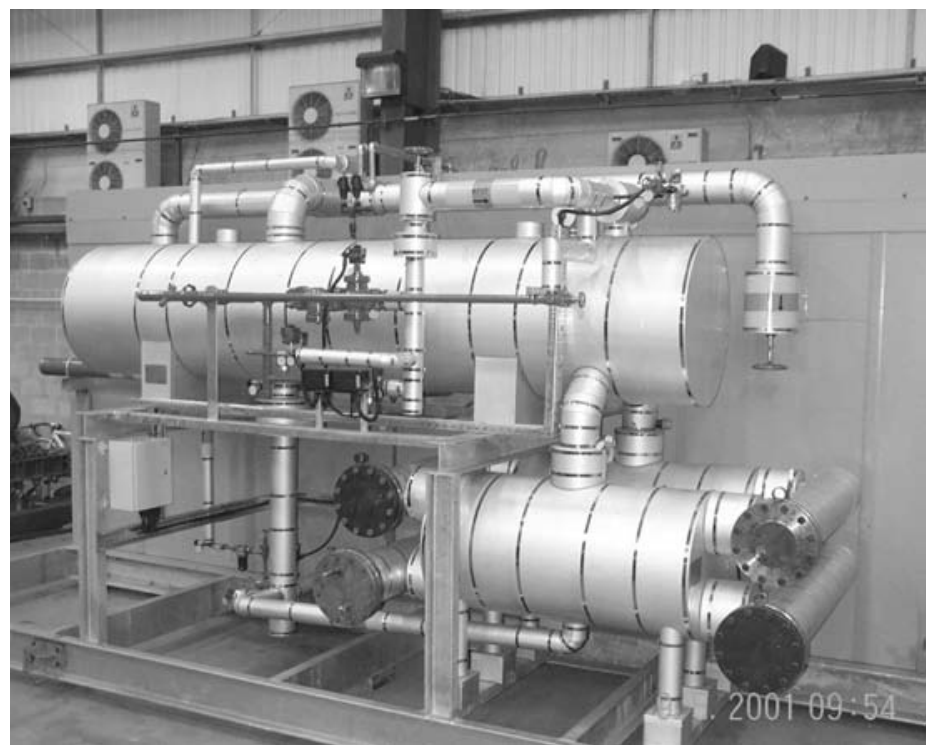

zentralen abgeführt. Dafür stehen drei unterschiedliche Temperaturniveaus zur Verfügung: $-5,-20$ und -45 Grad Celsius. Temperaturen von -45 Grad Celsius und tiefer werden ausserdem durch dezentrale Kaskaden-Kälteanlagen bereitgestellt, die ebenfalls an die allgemeine Versorgung angeschlossen sind. Durch die Fernkälteanlage mit mehreren grossen Einheiten erzeugt Bayer wirtschaftlich Kälte und gewährleistet eine kontinuierliche Versorgung.

In der York-Kältezentrale stellen zwei Schraubenverdichter-Aggregate Kälteenergie bereit. Das Kältemittel Ammoniak wird in kühlwasserbeaufschlagten Verflüssigern verflüssigt und über einen Unterkühler sowie bei Bedarf zuschaltbare Hochdruckpumpen in die Flüssigkeitsleitung zur Versorgung der Verbraucher eingespeist. Das aus dem Netz zurückkommende überhitzte Ammoniak-Gas wird in einem Saugabscheider abgekühlt und der Saugseite des Verdichters wieder zugeführt. Die Kälteleistung der Anlage beträgt über fünf Megawatt.

\section{Ammoniak-Anlage luftgekühlt}

Der dänische Arzneimittelhersteller Lundbeck Pharmaceuticals musste wegen gestiegener Nachfrage seine Fertigungskapazitäten im englischen Seal Sands ausbauen. Die Produktionsprozesse erfordern, dass genau vorgegebene Temperaturverläufe eingehalten werden. Dabei variieren die Temperaturen stark von -85 bis $+260 \mathrm{Grad}$
Celsius. Lundbeck suchte daher eine kältetechnische Lösung, die leistungsfähig, zuverlässig und umweltfreundlich ist - eine Herausforderung für Star Refrigeration.

Der Kälteanlagenbauer entwickelte zwei massgeschneiderte luftgekühlte AmmoniakSysteme, die Aufbau und Inbetriebnahme vor Ort reduzierten und jeweils einen Kreislauf mit Kälte versorgen. Der Normaltemperaturkreislauf bei +5 Grad Celsius kühlt stündlich $400 \mathrm{Ku}$ bikmeter des Kälteträgers Therminol D12. Die Kälteleistung beträgt 1,4 Megawatt. Im Tiefkühlkreislauf werden pro Stunde bei einer Kälteleistung

eurammon ist eine gemein same europäische Initiative von Unternehmen, Institutionen und Einzelpersonen, die sich für den verstärkten Einsatz von natürlichen Kältemitteln engagieren. Als Kompetenzzentrum für die Anwendung natürlicher Kältemittel in der Kältetechnik sieht die Initiative ihre Aufgabe darin, eine Plattform für Informationen und Austausch zu bieten und den Bekanntheitsgrad und die Akzeptanz natürlicher Kältemittel zu erhöhen. Ziel ist es, ihren Einsatz im welt $\mathrm{zu}$ fördern und so nachhaltiges Wirtschaften in der Kältetechnik weiterzuentwickeln. eurammon informiert Fachleute, Politiker und die breite Öffentlichkeit umfasInteresse einer gesunden Um- von 220 Kilowatt 50 Kubikmeter Therminol D12 auf -25 Grad Celsius gekühlt. In beiden Kreisläufen kommen schallisolierte Schraubenverdichter und leistungsfähige vollverschweisste Plate- and Shell-Verdampfer zum Einsatz, wodurch sich die Ammoniak-Füllmengen reduzieren lassen. Die Kältesysteme sind mit Speichern für die Trägerflüssigkeit ausgestattet. So können Ausfallzeiten überbrückt und Wartungen leichter vorgenommen werden. Star Refrigeration rüstete die Anlage mit moderner Steuerungstechnik einschliesslich Ammoniak-Detektoren aus. Auf Grund des modularen Designs kann der Betreiber die Installation jederzeit einfach und kosteneffizient erweitern.

\section{Prozesskühlung und Klima- tisierung aus einer Hand}

Dresen + Bremen realisierte für einen führenden deutschen Süsswarenhersteller eine Prozesskälteanlage, die mit Ammoniak betrieben wird. Auf seinem Werksgelände in Halle/Westfalen errichtete das Unternehmen ein neues Produktionsgebäude das mit einer Anlage für Prozesskühlung und Klimatisierung der Räumlichkeiten ausgestattet werden musste.

Nach einer detaillierten Wirtschaftlichkeitsbetrachtung mit Abwägung der anlagentechnischen Vor- und Nachteile, entschied sich der Betreiber für

send zu allen Aspekten natürlicher Kältemittel und steht allen Interessierten als kompetenter Ansprechpartner zur Verfügung. Anwendern und Planern von Kälteprojekten stellt eurammon konkrete Projekterfahrung sowie umfangreiches Informationsmaterial zur Verfügung und berät sie $\mathrm{zu}$ allen Fragen im $\mathrm{Zu}$ sammenhang mit Planung, Genehmigung und Betrieb von Kälteanlagen. Die Initiative wurde 1996 gegründet und steht europäischen Unternehmen und Institutionen im Interessenbereich natürlicher Kältemittel, aber auch Einzelpersonen beispielsweise aus Wissenschaft und Forschung offen.

Internetadresse:

www.eurammon.com 
eine Kälteanlage mit Ammoniak. Norbert Hackmann von Dresen + Bremen fasst die ausschlaggebenden Faktoren zusammen: «Die Anlage zeichnet sich durch einen niedrigen Energieverbrauch aus, ist auf Grund ihres redundanten Aufbaus sicher im Betrieb und hat sich in vier Jahren amortisiert. Ausserdem profitiert der Anwender vom positiven Image des natürlichen Kältemittels Ammoniak.»

Die Prozesskälte sorgt für die kontrollierte Wärmeabfuhr bei der Herstellung von Schokolade, Bonbons und Fruchtgummis sowie für die Kühlung der Maschinen. Herzstück der zen- tralen Anlage sind vier drehzahlgeregelte Schraubenverdichter. Die Verbraucher werden über zwei Flüssigkeitskreisläufe mit Temperaturen zwischen +5 und +11 Grad Celsius mit Kälte versorgt: In der Prozesskühlung zirkuliert Kaltwasser, während die Klimatisierung mittels Propylenglykol erfolgt. Plattenwärmetauscher sorgen für die Energieübertragung nach dem Schwerkraftprinzip. Die Verdampfungstemperaturen betragen jeweils +3 Grad Celsius, die Verflüssigungstemperaturen +33 Grad Celsius. Die Abwärme der Schraubenverdichter wird über einen ge- schlossenen Glykol-Kreislauf zur Erhitzung von Brauchwasser genutzt. Untergebracht ist die Kälteanlage in einem stählernen Maschinenraum auf dem Dach der Produktionshalle. Nach Abschluss des zweistufigen Ausbaus wird die Kälteleistung im Produktionsbereich knapp zwei Megawatt und im Klimabereich fast drei Megawatt betragen.

\section{Blick in die Zukunft}

«Grosse Potenziale sehen wir darüber hinaus bei der Erzeugung von Kälte in Bereichen von -30 bis -100 Grad Celsius beispielsweise in der chemischpharmazeutischen Industrie», erklärt abschliessend Georges Hoeterickx von eurammon. «Für diese Anwendungen stehen natürliche Kältemittel wie Kohlenwasserstoffe und Kohlendioxid als leistungsfähige und umweltfreundliche Alternativen bereit.»

\section{- eurammon}

Dr. Karin Jahn

Lyoner Strasse 18

D-60528 Frankfurt

Tel.: +49 (0)69 6603-1277

Fax: +49 (0)69 6603-2276

E-Mail:

karin.jahn@eurammon.com

Leserdienst Nr. 5

\section{Verpackungshersteller spart Geld durch Behandlung von Kühlturmwasser mit UV-Licht}

Seit der dänische Verpackungshersteller Styropack A/S ein UV-Desinfektionssystem zur Behandlung des Wassers in seinem offenen Kühlturm installiert hat, konnte das Unternehmen erhebliche Kosten einsparen. Das Kühlwasser wird für Kompressoren, Produktionsanlagen und einen Dampfkessel verwendet. - Bevor das Unternehmen auf UV umstieg, hatte es Probleme mit dem Wasserkühlsystem gegeben. Zunächst einmal setzten sich organisches Material und Bakterien auf den Lamellen* der Kühltürme ab. Die Lamellen mussten folglich alle sechs Monate gründlich gereinigt werden ein zeitaufwändiges und teures Verfahren. Bedingt durch die reduzierte Effizienz der Kühlturmlamellen liefen dann die Kühlturmventilatoren auf Hochtouren, wodurch wiederum die Stromrechnung des Unternehmens erheblich anstieg. Ein weiteres Problem bestand darin, dass mehr Füllwasser in der Anlage verbraucht wurde.

Styropack A/S versuchte zunächst, das Lamellen-Problem mit einem teuren Biozid zu lösen. Es stellte sich dabei jedoch als schwierig heraus, die richtige Dosis $\mathrm{zu}$ berechnen, und so wurde regelmässig überdosiert.

Schliesslich entschied sich das Unternehmen dafür, ein UVDesinfektionssystem zu testen, dessen Vorteile sofort zutage traten. Nach Absprache mit $\mathrm{HOH}$ $\mathrm{A} / \mathrm{S}$, Bersons dänischem Vertriebshändler, wurde ein bersonInLine ${ }^{\circledR}$-System mit einer bersonMultiWave ${ }^{\circledR}$ - Mitteldruck-

(*Lamellen: Rippen, die die Oberfläche für den Wärmeaustausch vergrößern)

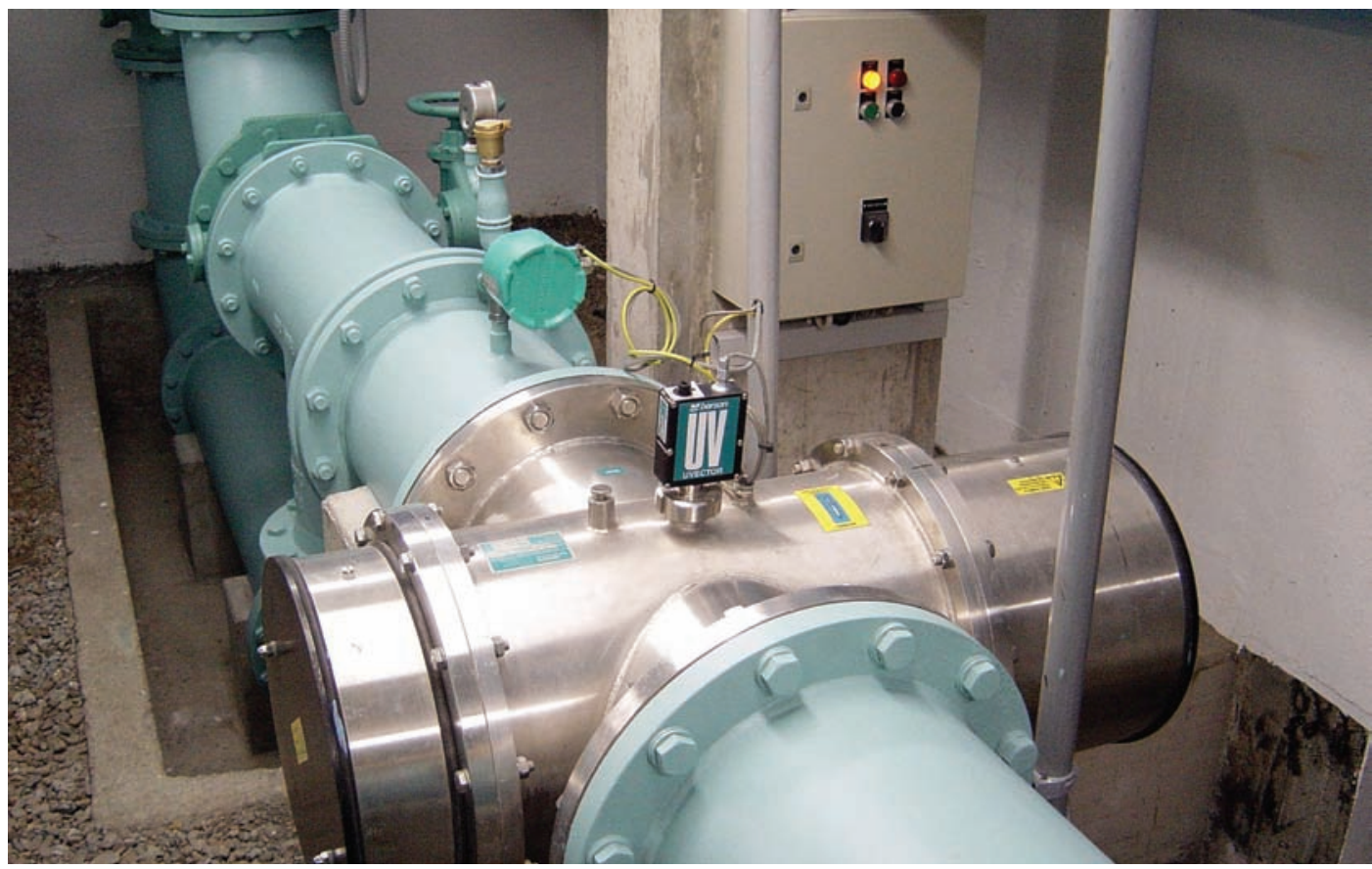

UV-Lampe installiert. Die bersonMultiWave ${ }^{\circledR}$-Lampe zerstört die DNS, RNS und Enzyme von Mikroorganismen und tötet Mikroorganismen dadurch irreversibel ab. Ein UV-Sensor überwacht die Aktivität der UVLampe und modifiziert bei Bedarf die UV-Dosis. Ausserdem sorgt ein automatischer Wischer dafür, dass der Quarzmantel, der die UV-Lampe umgibt, sauber bleibt. Die einzige erforderliche regelmässige Wartung besteht im jährlichen Auswechseln der UVLampe - ein einfacher Vorgang, der von Mitarbeitern vor Ort in wenigen Minuten erledigt wird.

Seit das UV-System vor etwas über einem Jahr installiert wurde, muss der Kühlturm nur noch jährlich im Rahmen der
Routinewartung inspiziert werden. Die Turmventilatoren laufen jetzt auch nur noch mit der minimal erforderlichen Leistung und es ist keine Biozid-Dosierung mehr erforderlich. Kosten wurden folglich drastisch gesenkt und das Wasser im offenen Kühlbecken ist jetzt so klar, dass man den Boden sehen kann.

Berson UV-techniek ist ein weltweit führendes Unternehmen für UV-Technologie für einen breiten Anwendungsbereich. Zum Leistungsspektrum des Unternehmens gehören die Aufbereitung von Prozesswasser, Abwasser, Trinkwasser und Schwimmbadwasser, Entchlorung, Entfernung von Ozon und TOC-Reduzierung. Der Sitz des Unternehmens befindet sich in den Niederlanden. Berson UVtechniek verfügt über mehr als 30 Jahre Erfahrung in der Konstruktion, Entwicklung, Herstellung und im Vertrieb von UVSystemen auf der ganzen Welt. Weitere Informationen befinden sich auf der Website des Unternehmens unter www.bersonuv.com.

- AQUATECH Solution GmbH Blegistrasse 15 6340 Baar

Tel.: 0417693111

Fax: 0417693121

E-Mail:

info@aquatech-solution.ch www.aquatech-solution.ch

Leserdienst Nr. 6 


\section{Introduction to Polarography and Voltammetry}

The latest work in the successful series of Metrohm Monographs offers a compact, easy-toread introduction to the basic principles of polar agraphic and voltammetric trace analysis. On not more than 60 pages the most important current measuring techniques are first described in a clear and illustrative manner. $\mathrm{Nu}-$ merous informative diagrams make it easier to understand the text. Direct current, pulse and alternating current methods are covered. The second main focus is on the different stripping voltammetry methods, which are among the most sensitive techniques available to the analytical chemist. Chapters on instrumentation and sample preparation in voltammetry round off the monograph. «Introduction to polarography and voltammetry» is intended for all those who wish to learn more about the basic principles of VA trace analysis This monograph is available in English and German. Just ask your local Metrohm distributor for a free copy.

- Metrohm Ltd.

PO Box

CH-9101 Herisau

Phone +41 713538585

Fax +41 713538901

www.metrohm.com

E-Mail:info@metrohm.com

Leserdienst $\mathrm{Nr} .7$

\section{pH mobile 826 - GLP/ISO jederzeit und überall}

Wo immer Sie pH-Werte messen möchten, das pH-mobile 826 ist $\mathrm{Ihr}$ zuverlässiger Begleiter. Basierend auf 60-jähriger Erfahrung in der $\mathrm{pH}$-Messung, liegt das $\mathrm{pH}$ mobile 826 voll auf der Linie der qualitativ hochstehenden Metrohm-Instrumente «Made in Switzerland». Wir verlassen uns aber nicht nur auf unsere Reputation, sondern sind bestrebt, Sie mit modernster Technologie zu verwöhnen:

\section{wipHi - drahtlose \\ Kommunikation mit \\ Drucker und PC}

- «Point and print» Anschluss an Drucker und PC via IrDaSchnittstelle

- Verschiedene GLP-konforme Reports und Ausdrucke

- Datentransfer an Metrodata VESUV 3.0 oder LIMS

\section{compHort - Bedienungs-} komfort durch Design

- Form und Funktion - modernes Design und hochwertige Materialien

- Einfache Bedienung - Cursorkontrollierter Menüdialog

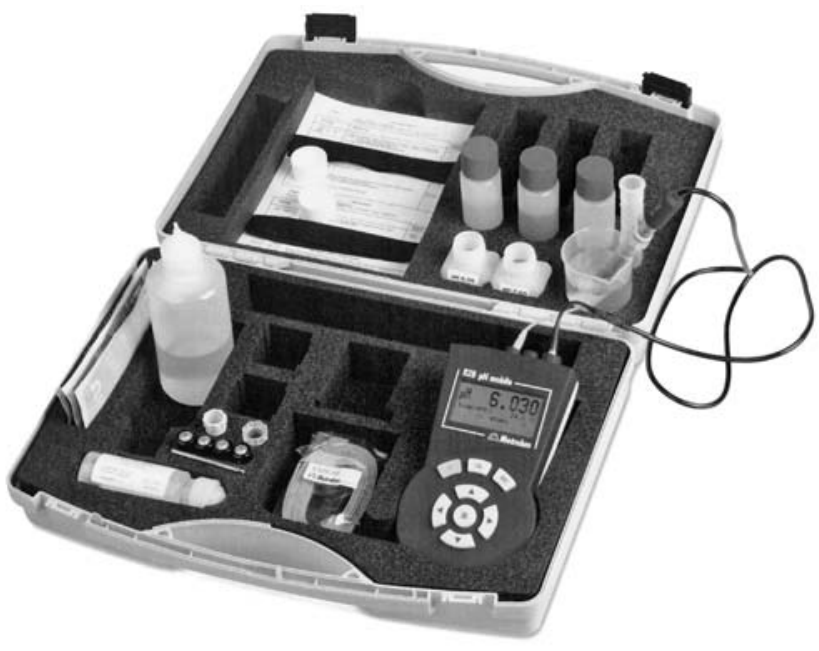

pHidelity - «Swiss made»Technologie, die Ihr Vertrauen verdient

- Hochpräziser Messeingang mit einer Auflösung von $0.001 \mathrm{pH}$

- GLP/ISO vom Besten: Dreipunkt-Kalibrierung mit automatischer PufferErkennung und Temperaturkompensation

- Anwender- und Probenidentifikation

- Resultatspeicher für 200 Messwerte (inkl. Datum, Zeit und Identifikation)
- Automatische Messwertübernahme nach Tastendruck, nach Erreichen einer Driftschwelle, nach einem vordefinierten Zeitintervall oder nach Erreichen einer vordefinierten Differenz zum vorausgegangenen Messwert

- Batteriebetrieb (4 x 1.5 V LR6 (UM3, AA), min. 500 h Betriebszeit)

- Schutzart IP67 (wenn Elektrode mit Stecker I verwendet wird)
Die neue «Long Life Primatrode» 6.0228.020 ist der ideale Sensor für das $\mathrm{pH}$ lab 826 . Zusammen mit dieser $\mathrm{pH}$ Elektrode erfüllt das Gehäuse des $\mathrm{pH}$ mobile 826 die Schutzart IP67 und erlaubt so den Einsatz in extrem feuchter Umgebung, wo mit Spritzwassereinwirkung $\mathrm{zu}$ rechnen ist. Auch wenn das Gerät bei der Messung mal ein unfreiwilliges Bad nehmen sollte, beeinträchtigt dies das Gerät nicht. Das $\mathrm{pH}$ mobile 826 ist auch in einem komplett ausgestattetem Transportkoffer erhältlich. Dieser enthält wichtige Utensilien wie z.B. Pufferlösungen, Messgefäss oder Probenfläschchen - kurz gesagt alles, was Sie für die Messung im Feld benötigen - und kann zudem auch als Arbeitsfläche benutzt werden.

- Metrohm AG

Postfach

9101 Herisau

Phone +4171353 8585

Fax +41713538901

www.metrohm.com

E-Mail:info@metrohm.com

Leserdienst $\mathrm{Nr} .8$

Für Ihre Werbung und Stellenangebote in CHIMIA:

KRETZ AG Verlag und Annoncen General Wille-Strasse 147, Postfach, CH-8706 Feldmeilen

Telefon 04492550 60, Fax 0449255077 